\title{
Alternative strategy for treating deep sternal wound infection following coronary artery bypass grafting with retention of the sternal plating system, systemic antibiotics, and vacuum assisted closure system: A case report
}

\author{
Fahad Athar ${ }^{1}$ and Louis Samuels ${ }^{2 *}$ \\ ${ }^{1}$ Division of Cardiothoracic Surgery, Albert Einstein Medical Center, Philadelphia, PA, USA \\ ${ }^{2}$ Department of Surgery, Thomas Jefferson University, Philadelphia, PA, USA
}

\begin{abstract}
Sternal wound infection (SWI) is an uncommon but potentially fatal complication of cardiac surgery following median sternotomy. It has a considerable impact on in-hospital morbidity and mortality, duration of hospital stay, mid-long term survival, and considerable financial concerns. The traditional approach to treating SWI involves removal of all foreign hardware, surgical debridement, and subsequent sternal reconstruction and long-term intravenous antibiotics. The therapy itself is associated with significant complications including chronic concerns for chest wall integrity, morbidity, and occasionally mortality. In this Case report, we describe an alternative strategy for deep sternal wound infection treatment that maintains sternal integrity by retention of the sternal plating system, soft tissue debridement, use of a vacuum-assisted closure system, and systemic antibiotics.
\end{abstract}

\section{Introduction}

Deep sternal wound infection (DSWI) is a complex and potentially devastating complication following cardiac surgery with a significant impact on both patient prognosis and hospital expenditure. The criteria defined by Center for Disease Control and Prevention (CDC) for DSWI must include at least one of the following within 90 days after the initial surgical procedure: organism cultured from mediastinal tissue or fluid obtained during a surgical operation or needle aspiration; evidence of mediastinitis seen during surgery; fever $\left(38^{\circ} \mathrm{C}\right)$, chest pain or sternal instability associated with purulent discharge from the mediastinal area or bacteria that is isolated from blood culture or culture of the mediastinal area [1]. Despite the poor clinical and economic outcomes of sternal wound infections, there are currently no specific guidelines in cardiac surgery to prevent and treat these infections. The purpose of this report is to describe an alternative approach that contrasts with the generally accepted principles of traditional practices.

\section{Case report}

A 51-year old woman with a past medical history of hypertension, hyperlipidemia and morbid obesity (BMI 41.2) presented with substernal chest pain for two weeks prior to admission. She also had a strong family history of premature death and coronary artery disease. Preoperative evaluation with echocardiograph and cardiac catherization discovered normal cardiac function, normal valvular function and severe multivessel coronary artery disease for which coronary artery bypass grafting (CABG) was recommended. Preoperative labs showed a hemoglobin of 13.3 , hematocrit of 40.5 , and normal electrolytes. Her albumin level was $3.2 \mathrm{~g} / \mathrm{ml}$ pre-operatively.

The patient was prepared for cardiac surgery under general anesthesia. She was given IV $1500 \mathrm{mg}$ of vancomycin and $2 \mathrm{~g}$ of
Cefazolin intra-operatively as part of the antibiotic prophylaxis protocol. Sternotomy was performed which was followed by open harvesting of left internal mammary artery (LIMA) and endoscopic harvesting of saphenous venous graft (SVG). The aorta and right atrium were cannulated for cardiopulmonary bypass (CPB). A normotensive $\mathrm{CPB}$ was initiated and a pump assisted beating heart five-vessel CABG was performed: SVG to Diagonal, Obtuse Marginal-1, Obtuse Marginal-2 and PDA, while LIMA to LAD. Ventilation was resumed and patient was weaned off $\mathrm{CPB}$ after a total time of 134 minutes. Trans-esophageal echocardiography showed good contractility and all grafts showed excellent flow on ultrasound probe. Chest tubes were placed in the pleural and mediastinal spaces. In view of her body habitus, closure was performed with the Sternalock $360^{\mathrm{mm}}$ (Zimmer Biomet; Warsaw, Indiana, U.SA). This sternal plating system uses titanium plates incorporated into titanium bands as well as titanium screws to hold the sternum together (Figure 1). The soft tissues were brought together using Vicryl suture and the overlying skin was approximated with a subcuticular suture.

The initial recovery was unremarkable. However, on POD 2, the patient developed a fever of $39^{\circ} \mathrm{C}$ with a leukocyte count of 16,000 . This was accompanied with a cloudy and purulent discharge from the incision site on POD 4. Due to the concern of sternal wound infection, she was empirically started on IV Piperacilling/Tazobactam and vancomycin. On POD 5, she received a Prevena ${ }^{\mathrm{T}}$ (Figure 2) wound management system (KCI Medical Ltd, Dublin, Ireland) that was applied atop the

*Correspondence to: Louis Samuels, Department of Surgery, Thomas Jefferson University, Philadelphia, PA, USA, E-mail: samuelsle@aol.com

Received: March 02, 2018; Accepted: March 26, 2018; Published: March 28, 2018 
Athar F (2018) Alternative strategy for treating deep sternal wound infection following coronary artery bypass grafting with retention of the sternal plating system, systemic antibiotics, and vacuum assisted closure system: A case report

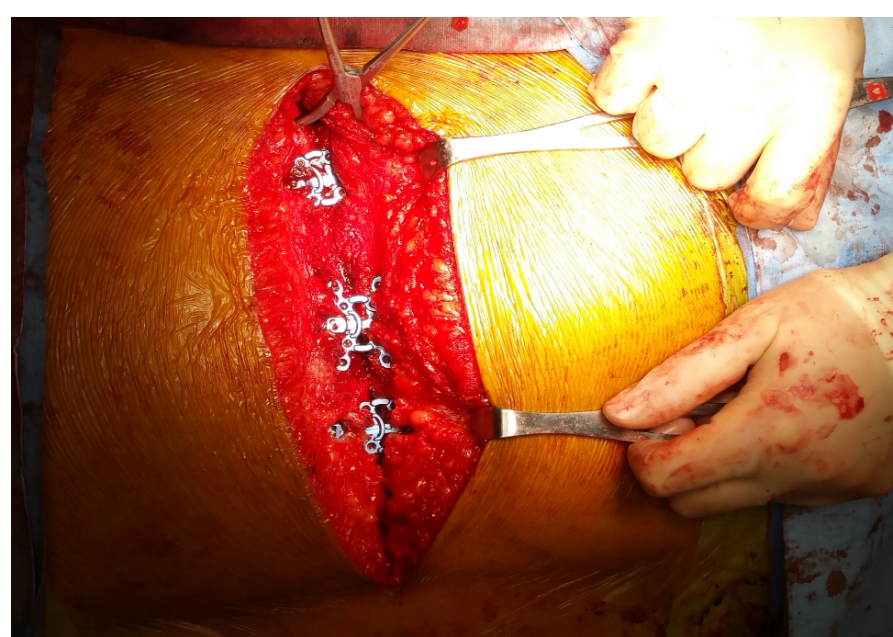

Figure 1. Sternal wound approximated with the Sternalock $360^{\mathrm{TM}}$ plating system

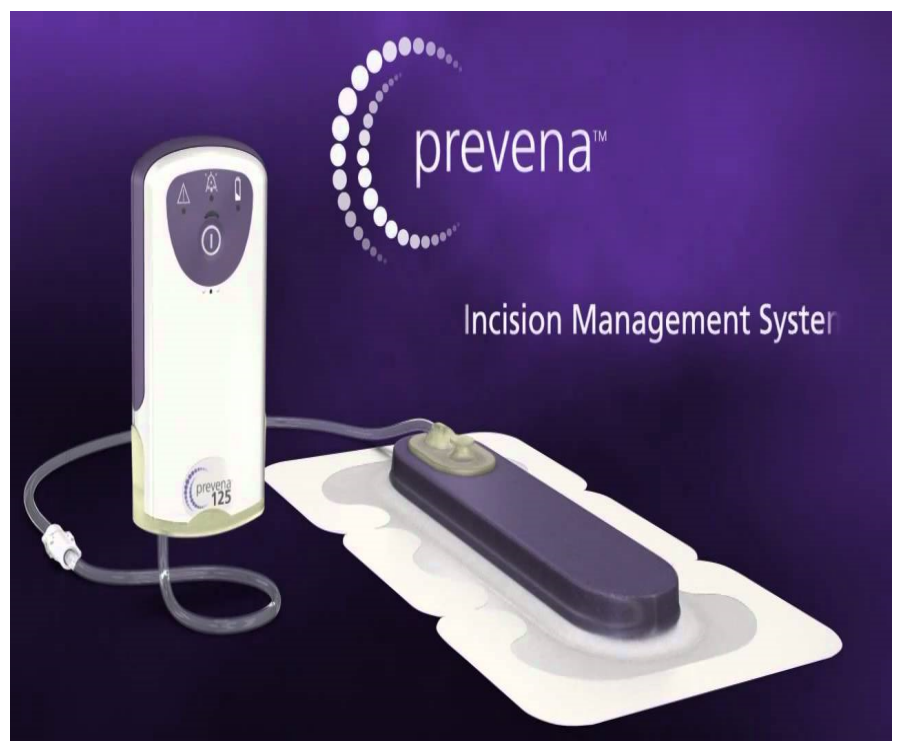

Figure 2. Prevena ${ }^{\mathrm{TM}}$ wound management system

sternal wound skin to absorb any secretions underneath. A culture of the discharge fluid was obtained at this time and showed no growth. Cultures from blood, urine and sputum were also negative for any growth. The initial impression was superficial fat necrosis.

On POD 7, due to persistent drainage, she was taken to the $\mathrm{OR}$ for sternal wound exploration. Intraoperative findings included fat necrosis associated with turbid fluid in proximity to the sternal bonecultures were obtained. The sternum itself remained well approximated, solid, and viable. A VAC device was applied (Figure 3). A culture was also obtained in the OR which again showed no growth.

She underwent bedside V.A.C $C^{\mathrm{m}}$ changes on POD 10 and POD 12 during which wound cultures were collected. The fevers had resolved and the wound appeared clean. On POD 14, she was taken to the OR where she underwent sternal soft tissue wound closure. Intraoperative cultures were obtained. On POD 15, she had another fever spike $\left(38.6^{\circ} \mathrm{C}\right)$. Cultures obtained previously now grew Serratia Marcescens. She was started on IV $2 \mathrm{~g}$ of Ceftriaxone every 24 hours after consultation with infectious disease specialists.

On POD 19, the patient underwent re-debridement of superficial soft tissues in conjunction with a V.A.C dressing in OR. Purulence was observed in the wound and the culture obtained in OR showed persistent growth of Serratia. V.A.C ${ }^{m}$ dressing changes continued throughout her stay at the hospital with no further attempts to close the sternal soft tissue. Again, the sternum itself appeared intact and solid with no indication of instability or dehiscence. Furthermore, despite the patient's large body habitus, the mechanics of breathing was unimpaired.

The patient continued to improve gradually as her fever and the leukocyte count returned to baseline. Follow-up cultures no longer showed any growth. The wound continued to appear clean with good granulation tissue with each V.A.C dressing change. She was transitioned to oral antibiotics (500 $\mathrm{mg}$ of Ciprofloxacin twice daily) upon discharge with frequent follow up in the surgical clinic. Frequent V.A.C. ${ }^{\mathrm{m}}$ dressing changes were done for two months while the soft tissue healed.

The remainder of the wound healing was uneventful with final reapproximation of the soft tissue assisted by the V.A.C. ${ }^{\text {ix }}$ system (Figure 4). Computer Tomography (CT) of the chest at this time showed the sternal segments to be closely approximated with no evidence of erosion or cortical destruction and without any soft tissue collections (Figure 5).

\section{Discussion}

Sternal wound infection is an uncommon outcome of cardiac surgery and is often divided into two categories: superficial and deep depending on the tissue involved. The superficial infections involve skin, sub-cutaneous tissue and the pectoralis fascia only without involvement of the bone. The incidence of superficial SWI is reported to range from $0.5-8 \%$ with a combined morbidity and mortality of 0.5 -
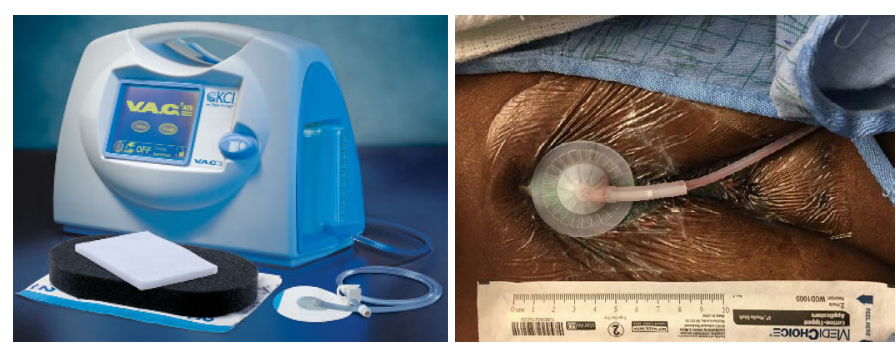

Figure 3. V.A.C. ${ }^{\text {тм }}$ System and its clinical application in this case

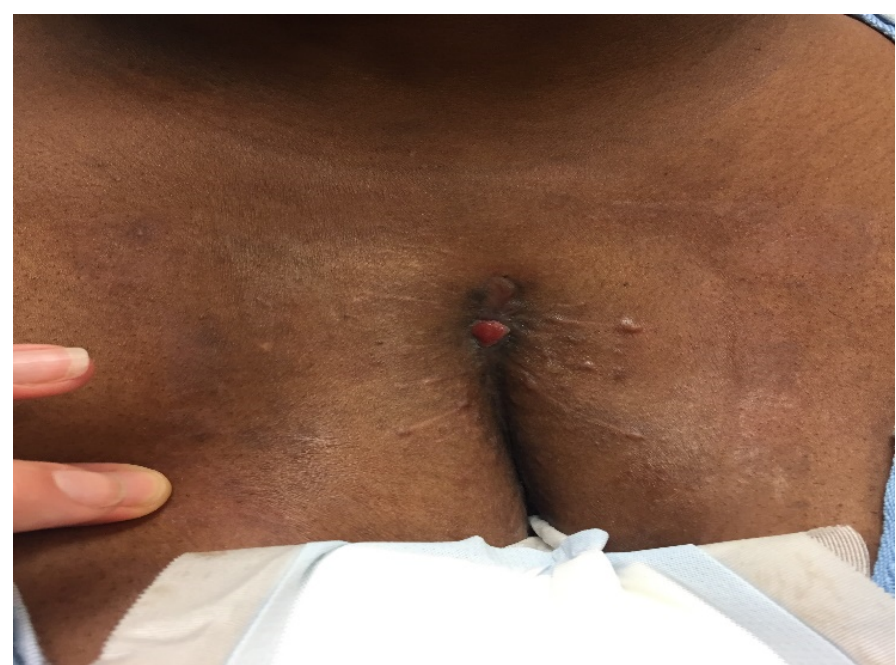

Figure 4. Sternal wound healed after prolonged treatment with V.A.C. ${ }^{\text {TM }}$ systemand antibiotics. 
Athar F (2018) Alternative strategy for treating deep sternal wound infection following coronary artery bypass grafting with retention of the sternal plating system, systemic antibiotics, and vacuum assisted closure system: A case report
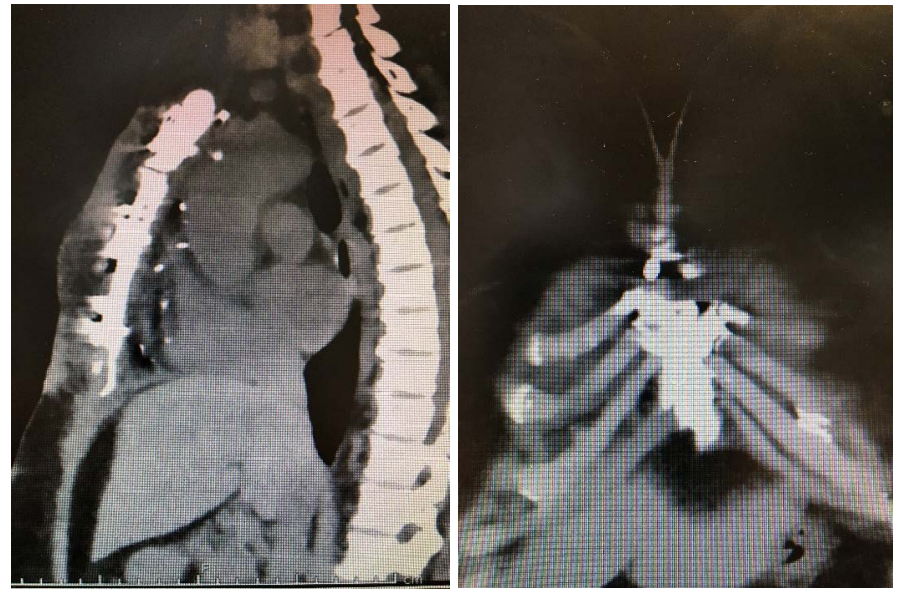

Figure 5. Saggital and Coronal sternal Computer Tomography following complete healing

$9 \%$ [2]. It is often diagnosed clinically by the presence of erythema, drainage, fever and occasional ternal instability; low-grade fever may be the only presenting complaint. Superficial SWI is often treated with IV antibiotics and local wound care.

Deep sternal wound infection (DSWI), also termed as mediastinitis, is a life-threatening complication after median sternotomy with an incidence of $1-5 \%$ and an associated mortality rate of $10-47 \%[3,4]$. Not only is it associated with poor clinical outcome but also with a longer duration of hospital stay and increased economic burden to the hospital. On average, the patients with DSWI spend an additional 20 days in the hospital as compared to those without DSWI after cardiac surgery and the cost for patients with sternal wound complications has been estimated to be 2.8 times that for the patients with uncomplicated post-operative courses [4,5]. Different studies suggested it could pose an additional cost ranging from $\$ 62,000$ to $\$ 500,000$ per case of post sternotomy mediastinits $[4,6]$.

Obesity is a known risk factor for DSWI, partly because of poor perfusion of subcutaneous adipose layers with low levels of prophylactic antibiotics into these tissues. Some data suggests a 1.5 to two-fold increase in DSWI after cardiac surgery in patients with body mass index $>30 \mathrm{~kg} / \mathrm{m}^{2}$ [7]. Other risk factors include age, chronic obstructive pulmonary disease, chronic cough, osteoporosis, re-operation, previous radiation therapy, previous myocardial infarction, bilateral mammary artery harvesting for bypass surgery, prolonged mechanical ventilation, smoking and peripheral vascular disease $[8,9]$. In addition, any factor that delays the healing can contribute to development to SWI. Malnutrition is a known risk factor and some studies have shown that patients with pre-operative hypoalbuminemia $(<3 \mathrm{~g} / \mathrm{ml})$ are more prone to develop DSWI [10]. Another study demonstrated that a preoperative albumin level of $<2.5 \mathrm{~g} / \mathrm{ml}$ was independently associated with both increased mortality and increased risk of developing SWI [11].

Despite advancement in prevention and perioperative care targeting to decrease the risk factors, DSWI still remains an alarming concern in cardiac surgery due to its short and long-term clinical and economic outcomes. The prophylactic antibiotic therapy is considered one of the most important preventive tools. However, the choice of antibiotic, the dose and the duration, the timing of prophylaxis and the optimum level required in serum and tissue still remains controversial [12].

Treating DSWI is often challenging and currently, the European and American cardiothoracic societies provide no guidelines for treating these infections. Classic principles of surgical wound infection includes debridement of infected and devitalized tissue as well as removal of any foreign bodies accompanied by antibiotics. The situation in cardiac surgery is compounded by the importance of the sternum from its coverage and protection of the underlying heart as well as its contribution to chest wall integrity and the mechanics of breathing. Morbidly obese patients have difficulty breathing on the basis of their body habitus such that any adverse condition affecting the mechanics of breathing in this patient population could be devastating. As such, the classical treatment of DSWI, which involves early wound exploration and extensive debridement [9], could prove fatal in some instances. Furthermore, removal of the the foreign material, such as sternal wires, may result in significant sternal instability with dire consequences. Traditionally, following extensive sternal wound debridement, sternal reconstruction is often achieved with the advancement of the pectoralis myocutaneous flap either as a single or staged procedure $[13,14]$. The procedure itself is associated with major adverse outcomes such as thoracic instability, prolonged immobilization with an increased risk of thrombosis, muscular weakness and pneumonia [15]. Another life-threatening complication includes right ventricular injury that can lead to uncontrolled tears with torrential bleeding and mortality [16]. These issues are compounded in the obese and morbidly obese patient population where tension on the midline of the sternum and its underlying scar tissue is higher than in their non-obese counterparts. The lateral tension associated with the obese patient has been studied and the forces in this area tend to pull the sternal halves apart [17]. In addition, the mechanics of breathing in the setting of sternal instability or absence of sternal integrity following its debridement may particularly problematic in patients with underlying COPD, as intrathoracic pressure is likely to be increased by the need for prolonged ventilator support with positive end expiratory pressures, as well as by excessive cough associated with COPD $[17,18]$. Again, these issues are compounded in the obese patient.

In our case, due to the high BMI, we elected to use a sternal plating system that combines titanium bands with plates and screws (SternaLock $360^{\mathrm{mm}}$ ) to re-approximate her sternum. This allows for a very secure sternal approximation related to its design of rigid fixation. In comparison to sternal wires, which provide a linear support, this plating system provides a two-dimensional support to stabilize the sternum [19]. In the obese and morbidly obese patients, the sternal wires may not be strong enough to hold the sternum together in periods of stress as when a large person coughs or engages in any activity that places lateral tension on the midline. It is not uncommon for sternal wires to pull through the hemi-sternums or to break altogether. In contrast, the design of the SternaLock $360^{\text {ma }}$ provides a much stronger closure that is not prone to break or pull through. In fact, some studies with sternal plates alone demonstrated a lower incidence of sternal infection in the group undergoing sternal plating compared that in those receiving conventional sternal wiring $[19,20]$. The system used in this case is unique because it adds the titanium bands together with the plates and screws.

Despite the development of a DSWI in this morbidly obese patient, two aspects are noteworthy: 1) the organism was a Serratia species, not the typical staphylococcus. Serratia infection is usually a water-born germ and its origin in this case is unclear. Nevertheless, once the species was identified, tailored antibiotic therapy was quickly utilized. 2) the infection clearly extended to the sternal hardware and was in contact with it and the bone. Despite this, we elected to retain the hardward and debride the necrotic and infected tissue, using the V.A.C. ${ }^{\text {rm }}$ to provide additional and frequent debridement while maintaining sternal bone integrity. We used the V.A.C ${ }^{m}$ due to its known advantages in causing 
Athar F (2018) Alternative strategy for treating deep sternal wound infection following coronary artery bypass grafting with retention of the sternal plating system, systemic antibiotics, and vacuum assisted closure system: A case report

a reduction in edema, removal of infectious secretions, stimulation of circulation by increasing arteriole dilation as well as granulation of soft tissues, thereby facilitating wound edge approximation and healing of soft tissue [21,22]. This translated into no deterioration in breathing mechanics. We were eventually able to transition the patient from inpatient to outpatient management. Vigilance in the outpatient clinic was important in the management of the wound and detection of any possible failure in this treatment plan that would require a more traditional approach. Fortunately, the wound healed and the patient was able to maintain a near normal lifestyle during the process.

Whether or not this approach is reproducible in similar patients remains to be seen. However, the merits of this approach are several, but most notably is sternal integrity. In our opinion, the decisions described in this case may be worthwhile as a first-line approach for sternal wound infections in the obese and morbidly obese patient population since there is little tolerance for any disruptions in the mechanics of breathing. In addition, avoidance of sternal bone resection, which is sometimes necessary, may reduce or eliminate complications associated with this hazardous operation, particularly bleeding.

In summary, we describe an approach to the treatment of deep sternal wound infection that utilizes technologies that maintain sternal integrity and treat devitalized and infected soft tissue. These technologies, in combination with systemic antibiotics and vigilance in wound care may be superior to the traditional approach. Further application and examination is warranted.

\section{References}

1. Garner JS, Jarvis WR, Emori TG, Horan TC, Hughes JM (1988) CDC definitions for nosocomial infections, 1988. Am J Infect Control 16: 128-140. [Crossref]

2. Ridderstolpe L, Gill H, Granfeldt H, Ahlfeldt H, Rutberg H (2001) Superficial and deep sternal wound complications: incidence, risk factors, and mortality. Eur J Cardiothorac Surg 20: 1168-75.

3. Gummert JF1, Barten MJ, Hans C, Kluge M, Doll N, et al. (2002) Mediastinitis and cardiac surgery - an updated risk factor analysis in 10,373 consecutive adult patients. Thorac Cardiovasc Surg 50: 87-91. [Crossref]

4. Singh K, Anderson E, Harper JG (2011) Overview and management of sternal wound infection. Semin Plast Surg 25: 25-33. [Crossref]

5. Losanoff JE, Richman BW, Jones JW (2002) Disruption and infection of median sternotomy: a comprehensive review. Eur J Cardiothorac Surg 21: 831-839. [Crossref]

6. Lazar HL, Salm TV, Engelman R, Orgill D, Gordon S (2016) Prevention and management of sternal wound infection. J Thorac Cardiovasc Surg 152: 962-72. [Crossref]
7. Cotogni P, Barbero C, Rinaldi M (2015) Deep sternal wound infection after cardiac surgery: Evidences and controversies. World J Crit Care Med 4: 265-273. [Crossref]

8. Lu JC1, Grayson AD, Jha P, Srinivasan AK, Fabri BM (2003) Risk factors for sternal wound infection and mid-term survival following coronary artery bypass surgery. Eur J Cardiothorac Surg 23: 943-949. [Crossref]

9. Rupprecht L, Schmid C (2013) Deep Sternal Wound Complications: An Overview of Old and New Therapeutic Options. Open J Cardiovasc Surg 6: 9-19. [Crossref]

10. Rady MY, Ryan T, Starr NJ (1997) Clinical characteristics of preoperative hypoalbuminemia predict outcome of cardiovascular surgery. J Parenter Enteral Nutr 21: 81. [Crossref]

11. Engelman DT, Adams DH, Byrne JG, Ranki SF, Collins JJ, et al. (1999) Impact of body mass index and albumin on morbidity and mortality after cardiac surgery. $J$ Thorac Cardiovasc Surg 118: 866-71. [Crossref]

12. Edwards FH, Engelman RM, Houck P, Shahian DM, Bridges CR (2006) The Society of Thoracic Surgeons Practice Guideline Series: Antibiotic Prophylaxis in Cardiac Surgery, Part I: Duration. Ann Thorac Surg 81: 397-404. [Crossref]

13. Fleck TM, Fleck M, Moidl R, Czerny M, Koller R, et al. (2002) The vacuum-assisted closure system for the treatment of deep sternal wound infections after cardiac surgery. Ann Thorac Surg 74: 1596-1600. [Crossref]

14. Wong CH, Senewiratne S, Garlick B, Mullany D (2006) Two-stage management of sternal wound infection using bilateral pectoralis major advancement flap. Eur $J$ Cardiothorac Surg 30: 148-152. [Crossref]

15. Schimmer C1, Sommer SP, Bensch M, Leyh R (2007) Primary treatment of deep sternal wound infection after cardiac surgery: a survey of German heart surgery centers. Interact Cardiovasc Thorac Surg Volume 6: 708-711. [Crossref]

16. Sjogren J, Malmsjo M, Gustafsson R, Ingemansson R (2006) Poststernotomy mediastinits: a review of conventional surgical treatments, vacuum-assisted closure therapy and presentation of Lund university hospital mediastinits algorithm. Euro $J$ cardiothoracic Surg 30: 895-905. [Crossref]

17. Bitkover CY, Gårdlund B (1998) Mediastinitis after cardiovascular operations: a casecontrol study of risk factors. Ann Thorac Surg 65: 36-40. [Crossref]

18. Bryan AJ, Lamarra M, Angelini GD, West RR, Breckenridge IM (1992) Median Sternotomy wound dehiscence: a retrospective case control study of risk factors and outcomes. J R Coll Surg Edinb 37: 305-308. [Crossref]

19. Hirose H, Yamane K, Youdelman BA, Bogar L, Diehl JT (2011) Rigid Sternal Fixation Improves Postoperative Recovery. Open Cardiovasc Med J 5: 148-152. [Crossref]

20. Raman J, Song DH, Bolotin G, Jeevanandam V (2006) Sternal closure with titanium plate fixation--a paradigm shift in preventing mediastinitis. Interact Cardiovasc Thorac Surg 5: 336-9. [Crossref]

21. Wackenfors A, Gustafsson R, Sjogren J, Algotsson L, Ingemansson R, et al. (2005) Blood flow responses in the peristernal thoracic wall during vacuum-assisted closure therapy. Ann Thorac Surg 79: 1724-1730. [Crossref]

22. Lambert KV, Hayes P, McCarthy M (2005) Vacuum assisted closure: a review of development and current applications. Eur J Vasc Endovasc Surg 29: 219-226. [Crossref]

Copyright: (C2018 Athar F. This is an open-access article distributed under the terms of the Creative Commons Attribution License, which permits unrestricted use, distribution, and reproduction in any medium, provided the original author and source are credited. 